\title{
Implementación de la Metodología ABP (Aprendizaje Basado en Problemas) en Estudiantes de Primer año de la Carrera de Educación Diferencial
}

\author{
Implementation of PBL Methodology (Problem Based Learning) in First Year Students of \\ the School of Special Education
}

\author{
Aplicação da Metodologia PBL (Problem Based Learning) em alunos do primeiro ano \\ da Escola de Educação Especial
}

\section{Cecilia Jofré $M .^{\mathrm{a}}$ y Fernando Contreras $H^{\mathrm{b}}$}

${ }^{a}$ Escuela de Educación Diferencial, Universidad San Sebastián. Correo electrónico: cecilia.jofre@uss.cl. Telf.: (56)(41)2400293

bUniversidad San Sebastián. Correo electrónico: fernando.contreras@uss.cl. Telf.: (56)(41)2400304

\begin{abstract}
RESUMEN
En educación superior son variadas las metodologías que se proponen para cumplir con las exigencias en la formación de profesionales. En este escenario, se desarrolla el proyecto de Aprendizaje Basado en Problemas (ABP) para ser implementado en estudiantes de primer año de Pedagogía en Educación Diferencial. De los resultados obtenidos se puede concluir que los estudiantes alcanzaron un nivel de desempeño acorde a los objetivos del proyecto. Esto en base a las calificaciones finales ponderadas $(\mathrm{ME}=6.2, \mathrm{DS}=0.44)$, las cuales dan cuenta de un buen desarrollo de la metodología ABP. Además, los estudiantes concuerdan al señalar que lograron conocer y aplicar una estrategia general de resolución de problemas que puede ser utilizada en contextos reales.
\end{abstract}

Palabras clave: aprendizaje basado en problemas, educación superior, metodología de enseñanza.

\begin{abstract}
In higher education there are various methodologies proposed to fulfill the goals of the professional training. The project "Problem Based Learning" (PBL) was developed in this scenario and implemented in the first year of Special Education Pedagogy Program. Considering the results obtained, it was concluded that the level of performance achieved by students was in agreement with the objectives of the project. This conclusion is based on weighed final grades $(\mathrm{ME}=6.2, \mathrm{SD}=0.44)$, which account for a good development of the PBL methodology. In addition, students agree in stating that they were able to know and apply a comprehensive strategy for solving problems which can be used in real contexts.
\end{abstract}

Key words: problem-based learning, higher education, teaching methodology.

\section{RESUMO}

Na Educação Superior são várias as metodologias propostas para atender as demandas da formação de profissionais. Neste cenário, desenvolve-se o projeto de Aprendizagem Baseada em Problemas (ABP), a ser implementado em estudantes do primeiro ano da Graduação em Pedagogia da Educação Especial. A partir dos resultados obtidos, conclue-se que os estudantes alcançaram um nível de desempenho compatível com os objetivos do projeto. Isto conforme as notas finais ( $\mathrm{ME}=6,2, \mathrm{DS}=0,44)$, as quais dão conta de um bom desenvolvimento da metodologia ABP. Além disso, os estudantes concordam ao afirmar que conseguiram e aplicar uma estratégia global de resolução de problemas que pode ser usadas em contextos reais.

Palavras chave: aprendizagem baseada em problemas. educação superior. metodologia de ensino. 


\section{INTRODUCCIÓN}

En la actualidad, los cambios asociados a la denominada sociedad de la información exigen modificaciones en los modelos educativos, cambios en los usuarios de la educación y cambios en los escenarios donde tiene lugar el proceso de aprendizaje. Estas modificaciones en educación apuntan al desarrollo de competencias en los estudiantes, las cuales se reconocen como críticas para un adecuado desempeño en la sociedad. De esta forma, se ha tomado conciencia de que el desarrollo de competencias básicas constituye un elemento central para el aprendizaje (Slavin, 2008).

El desarrollo de destrezas y competencias individuales en contextos formales se ve beneficiado por la interacción con otros. No basta sólo con presentar determinadas actividades de aprendizaje, es necesario organizar el contexto en el cual el aprendizaje ocurre con el fin de optimizar el proceso y potenciar las destrezas de los alumnos (Arens, 2005). En este sentido, en la búsqueda de un método didáctico generador de conocimientos significativos y de habilidades, se presenta el Método de Aprendizaje Basado en Problemas (ABP) como una alternativa efectiva para ser implementada en carreras de Pedagogía.

En este escenario y como parte del proyecto MECESUP implementado en el año 2008 en la Universidad San Sebastián, que se orienta al diseño de un sistema permanente de nivelación y fortalecimiento de competencias básicas para los estudiantes de Primer año de las carreras de la Facultad de Educación, se desarrolla en el mes de Noviembre de 2010 en la carrera de Pedagogía en Educación Diferencial el Proyecto de Aprendizaje Basado en Problemas.

Al aplicar el ABP como estrategia metodológica en el aula, se ha observado que los estudiantes desarrollan habilidades como la comprensión lectora, capacidad de análisis, síntesis y relación de información, entre otras. Estas habilidades se potencian debido a que deben lograr identificar una problemática inmersa en la redacción del caso-problema. Luego de identificar una problemática, tienen que analizar y sintetizar los aportes de cada miembro del grupo de trabajo y, posteriormente, deberán formular una hipótesis que permita explicar el problema y, de esta forma, establecer acciones remediales, las cuales deben ser evaluadas para seleccionar la mejor estrategia de solución al problema (Coll, Mauri \& Onrubia, 2006).

Adicionalmente, los estudiantes requieren del uso de estrategias de búsqueda, selección y análisis de información que permita realizar una adecuada descripción y comprensión del problema identificado, así como también de las hipótesis y estrategias de solución elaboradas (Woodfolk, 2006).

El proyecto ABP está diseñado en base a talleres grupales, asesorados y dirigidos por docentes de los cursos que se dictan en $1^{\circ}$ año y docentes de planta en el rol de facilitadores. Estos talleres se desarrollaron durante una semana donde la totalidad de los módulos de cada asignatura se orientó al trabajo de los estudiantes. Se definieron grupos de trabajo, en los cuales, a través de un abordaje práctico y progresivo, se implementó la metodología de Aprendizaje Basado en Problemas.

La evaluación se realizó utilizando diversas pautas e instructivos y, como actividad de cierre, los estudiantes presentaron los trabajos realizados por los distintos grupos, promoviendo en todo momento el desarrollo de habilidades investigativas y sociales. 


\section{OBJETIVOS}

\section{Objetivo General}

1.- Desarrollar talleres prácticos para los estudiantes de primer año de la carrera de Pedagogía en Educación Diferencial en base al método de Aprendizaje Basado en Problemas.

2.- Favorecer el desarrollo de habilidades y destrezas sociales, actitudinales y cognitivas para la búsqueda, análisis, integración y transferencia de conocimientos.

\section{Objetivos Específicos}

1.- Incorporar la metodología de Aprendizaje Basado en Problemas como estrategia transversal en asignaturas de especialidad de la Carrera de Educación Diferencial y en las prácticas progresivas y práctica profesional.

2.- Desarrollar estrategias y métodos que permitan ser aplicados y transferidos en el futuro a situaciones semejantes para la resolución de problemas.

3.- Favorecer el aprendizaje autodirigido y el desarrollo de habilidades de comunicación en los estudiantes.

4.- Desarrollar la capacidad de trabajar en equipo y en redes a través de la posesión de actitudes y disposiciones que incluyen la flexibilidad y adaptabilidad.

5.- Estimular el pensamiento crítico en los estudiantes.

Metas Propuestas

Se establece en el proyecto como metas a lograr las siguientes:

1.- Incorporación en $1^{\circ}$ año de la carrera de Pedagogía en Educación Diferencial del Taller de Aprendizaje Basado en Problema.

2.- Proporcionar, al $100 \%$ de los de los estudiantes de $1^{\circ}$ año de la Carrera, una herramienta metodológica de aprendizaje que facilitará la búsqueda de la solución de problemas a las que deberán enfrentarse en su quehacer como estudiantes y miembros de la sociedad.

3.- Capacitar a los Docentes especialistas en Educación Diferencial en la metodología de Aprendizaje basado en problemas para su utilización en las diversas tareas y actividades programadas en las asignaturas de la especialidad.

4.- Aplicación del Método de Aprendizaje basado en problemas en los trabajos de las asignaturas y módulos de la especialidad de la Carrera de Pedagogía en Educación Diferencial.

El desarrollo e implementación del proyecto abarcó durante el año 2010 las primeras tres metas, quedando para su incorporación como estrategia metodológica en las asignaturas de especialidad para el año académico de 2011.

\section{DESCRIPCIÓN E IMPLEMENTACIÓN}

\subsection{ACTIVIDADES DESARROLLADAS}

Para el desarrollo del proyecto ABP se estableció un cronograma de actividades tendientes a asegurar su exitosa implementación. Estas actividades se encuentran definidas en el proyecto y se resumen en la Tabla 1: 
Tabla 1. Actividades Proyecto ABP

\begin{tabular}{|c|c|}
\hline Actividades & Tareas \\
\hline $\begin{array}{l}\text { 1. Docentes diseñan evaluaciones y organizan } \\
\text { talleres. }\end{array}$ & $\begin{array}{l}\text { - Organizar el trabajo de los docentes. } \\
\text { - Diseño de pautas de evaluación: } \\
\text { heteroevaluación, coevaluación y } \\
\text { autoevaluación. }\end{array}$ \\
\hline 2. Reunión con los docentes, decano. & - Presentación del Proyecto. \\
\hline $\begin{array}{l}\text { 3. Reuniones de capacitación a docentes de la } \\
\text { carrera de Educación Diferencial en cada una } \\
\text { de sus sedes. }\end{array}$ & $\begin{array}{l}\text { - Presentación del proyecto. } \\
\text { - Planificación de reuniones de capacitación. }\end{array}$ \\
\hline $\begin{array}{l}\text { 4. Presentación de la calendarización y } \\
\text { organización de los talleres. }\end{array}$ & • Acopio de la información. \\
\hline 5. Organización de los talleres tutoriales. & $\begin{array}{l}\text { - Determinar los dos docentes que participarán } \\
\text { en los talleres tutoriales. } \\
\text { - Distribución de los grupos para trabajo en los } \\
\text { talleres tutoriales. }\end{array}$ \\
\hline $\begin{array}{l}\text { 6. Implementación y ejecución de los talleres } \\
\text { con los estudiantes de } 1^{\circ} \text { año de la Carrera de } \\
\text { Pedagogía en Educación Diferencial. }\end{array}$ & $\begin{array}{l}\text { - Realización de los Talleres con los } \\
\text { estudiantes de } 1^{\circ} \text { año. } \\
\text { - Aplicación de pautas de evaluación. }\end{array}$ \\
\hline $\begin{array}{l}\text { 7. Reuniones de los docentes, análisis } \\
\text { y evaluación del logro de productos y } \\
\text { consecución de objetivos del proyecto. }\end{array}$ & $\begin{array}{l}\text { - Organización de reunión. } \\
\text { - Aplicación pauta de evaluación del taller } \\
\text { Aprendizaje basado en problemas. }\end{array}$ \\
\hline
\end{tabular}

La mayor parte de las actividades programas se realizaron durante el primer semestre de 2010 con excepción de las actividades 3, 6 y 7, las cuales fueron desarrolladas en el segundo semestre.

Las actividades asociadas al proceso de organización de los talleres incluye el diseño de los casos-problema que fueron utilizados en el trabajo con los estudiantes, así como también la metodología para la conformación de los grupos y la elaboración del cronograma de actividades a realizar en la implementación del proyecto.

Los casos-problema se diseñaron utilizando como marco de referencia las propuestas derivadas de la metodología de Análisis de Casos. De esta forma, los casos corresponden a una descripción de una situación real y concreta que los estudiantes, de manera cooperativa, deben someter a un análisis y a la toma de decisiones. Los casos utilizados en el proyecto presentan las siguientes características (Mucchielli, 1970):

1. Autenticidad: El caso corresponde a una situación concreta y basada en el contexto real, por tanto es creíble para los estudiantes.

2. Urgencia de la Situación: Presenta una situación problemática que exige la toma de decisiones o implementación de una solución.

3. Orientación Pedagógica: La situación presentada permite al estudiante la aplicación de lo aprendido en un área o áreas específicas, siendo el contexto para transferir los conocimientos a una realidad concreta. 
4. Totalidad: El caso contiene la información necesaria y suficiente para la realización del proceso de análisis y posterior toma de decisiones.

En el desarrollo e implementación de esta metodología se establece un proceso de guía docente a los estudiantes. Este acompañamiento sigue las directrices señaladas tanto en el método ABP como en el Análisis de Casos. Estos lineamientos se resumen en la Figura 1.

Figura 1. Esquema Resumen Construcción y Análisis de Casos

\begin{tabular}{|c|c|c|c|c|c|}
\hline$\vec{~}$ & $\begin{array}{l}\text { Proceso de } \\
\text { Elaboración. } \\
\text { Especificar } \\
\text { Objetivos. } \\
\text { Búsqueda de } \\
\text { Información. } \\
\text { Elaboración } \\
\text { de Preguntas. }\end{array}$ & 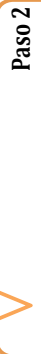 & $\begin{array}{l}\text { Presentación de } \\
\text { los objetivos. } \\
\text { Conformación } \\
\text { de grupos. } \\
\text { Presentación del } \\
\text { caso. } \\
\text { Espacio para } \\
\text { inquietudes y } \\
\text { guía de análisis. }\end{array}$ & $\begin{array}{l}m \\
\mathscr{8} \\
\tilde{g} \\
\tilde{g}\end{array}$ & $\begin{array}{l}\text { Discusión del } \\
\text { Caso. } \\
\text { Debate general } \\
\text { guiado por el } \\
\text { Docente. } \\
\text { Adopción de } \\
\text { una solución por } \\
\text { consenso y } \\
\text { Síntesis de las } \\
\text { aportaciones } \\
\text { Realizadas. }\end{array}$ \\
\hline
\end{tabular}

De esta forma, el trabajo realizado por los estudiantes constituye una combinación del método ABP y el Análisis de Casos en base a la Figura 2:

Figura 2. Esquema Resumen Secuencia ABP

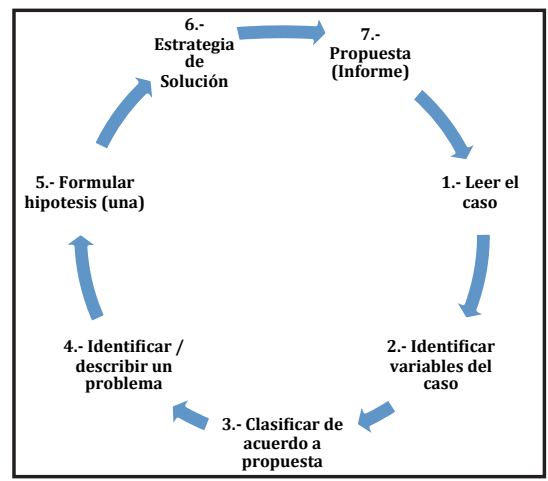

La secuencia de acciones sigue una progresión desde la actividad inicial de lectura del caso hasta la presentación de una propuesta de solución que queda registrada en un informe. En cada etapa de esta secuencia los estudiantes reciben la orientación de un docente, la cual actúa como catalizador del proceso de andamiaje, favoreciendo el aprendizaje (Kingler \& Vadillo, 2003).

Para implementar la secuencia descrita en la Figura 2 se estableció un cronograma de actividades para cada día de la semana en la que se desarrolló el proyecto. Este cronograma responde a la necesidad de entregar una estructura clara y definida a los estudiantes, facilitando el proceso de guía por parte de los docentes y permitiendo organizar el trabajo en forma más autónoma. 
La conformación de los grupos de trabajo se realizó utilizando como criterio el azar. De esta forma, se constituyeron 10 grupos integrados por 11 estudiantes cada uno. Esta información fue entregada a los estudiantes a través del portal de la Universidad la semana anterior a la realización del proyecto ABP.

Previo al inicio de las actividades, se realizó una presentación a los estudiantes del cronograma de actividades, de las pautas de evaluación y se les entregó un horario donde se indicaba los diferentes momentos y los docentes que se reunirían con ellos para trabajar. Este incluía un módulo (de 80 minutos) diario obligatorio de trabajo por grupo guiado por un docente y especificaba el horario para consultas, el resto del tiempo era de administración de cada grupo. En esta instancia se entregó el portafolio de trabajo a cada grupo y las carpetas a los docentes (Figura 3), además permitió resolver dudas de los estudiantes respecto a algunos aspectos formales como asistencia y calificación.

En la primera reunión tutorial, los grupos se organizaron asignando los siguientes roles: Líder del grupo, secretario 1 (registro de funcionamiento del grupo), secretario 2 (registro de información) y un coordinador.

Figura 3. Contenido Portafolios y Carpeta Docente

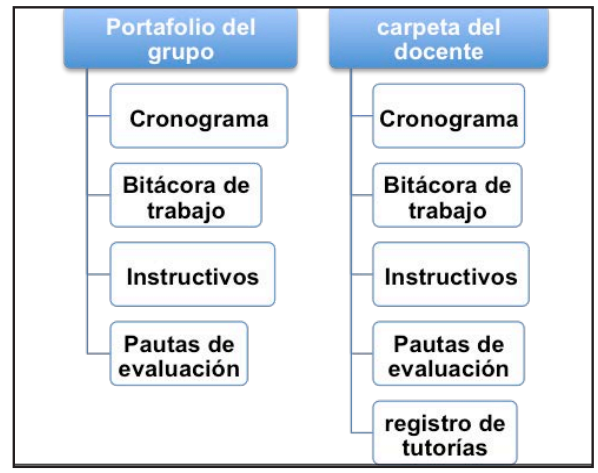

Para el análisis del caso, se entregó a los estudiantes una plantilla de clasificación de factores para facilitar la reflexión y comprensión de la situación-problema (Figura 4).

Figura 4. Planilla Clasificación para Análisis de Casos

\begin{tabular}{|c|c|c|}
\hline \multirow{2}{*}{ CATEGORÍA } & SUBCATEGORÍA & $\begin{array}{c}\text { FACTOR } \\
\text { PRESENTE EN EL } \\
\text { CASO }\end{array}$ \\
\hline \multirow{2}{*}{$\begin{array}{c}\text { FACTORES NO } \\
\text { PERSONALES }\end{array}$} & SOC. GENERAL & \\
\cline { 2 - 3 } & SOC. ESCOLAR & \\
\cline { 2 - 3 } & SOC. FAMILIAR & \\
\hline \multirow{3}{*}{ FACTORES } & FÍSICOS & \\
\cline { 2 - 3 } PERSONALES & PSICOLÓGICOS Y & \\
& PEDAGÓGICOS & \\
\cline { 2 - 3 } & RELACIONALES & \\
\hline
\end{tabular}


Desde el inicio, se entregó las diferentes rúbricas que se utilizarían para la evaluación de los estados de avance: a) Identificación y descripción del problema, b) formulación de la hipótesis con su mapa conceptual y marco teórico referencial y c) estrategias de solución. Se informó a los estudiantes de las exigencias y formato de los estados de avance y del informe final.

En relación a la presentación oral de los estados de avance, no siempre fueron realizados de acuerdo a lo programado, debido a la dinámica de funcionamiento de los grupos y, principalmente a la imposibilidad de asignar un tutor único para cada grupo debido al bajo número de docentes de planta. En este sentido, se optó por suplir esta falencia con docentes part-time, los cuales solo podían participar en los horarios comprometidos en sus asignaturas y en horarios fuera de la programación. Esto se traduce en rotación de docentes en los grupos. Como consecuencia de lo anterior, fue frecuente el hecho de que los docentes, al trabajar con el grupo, desconocían el trabajo realizado previamente, por lo cual destinaban los primeros 10 o 15 minutos a informarse del estado de avance del grupo y de las acciones realizadas. Esta dinámica de funcionamiento, si bien podría haber sido un obstáculo, permitió a los estudiantes reflexionar respecto de sus análisis, introducir nuevas interrogantes o perspectivas de análisis que se desprendían de los comentarios de los docentes que rotaban por los grupos, lo cual se traduce en mejoras en la calidad de los productos presentados.

\section{TÉCNICAS E INSTRUMENTOS}

Para el proceso de evaluación del proyecto y su implementación se utilizaron la técnica de cuestionario y observación. La elección de estas técnicas responde a los objetivos del proyecto y a la necesidad de obtener información del proceso y dinámica de funcionamiento de cada uno de los grupos de estudiantes.

Se evaluaron los estados de avance y los productos finales del proyecto (informe y exposición final), proceso a cargo de docentes de planta de la carrera, para lo cual se utilizaron pautas de observación y rúbricas de desempeño. Además, se utilizó la estrategia de autoevaluación y heteroevaluación, las cuales fueron en base a cuestionarios con preguntas abiertas y listas de cotejo.

El proceso total de evaluación se resume en la Tabla 2 que se presenta a continuación:

Tabla 2. Evaluación ABP

\begin{tabular}{|c|c|c|c|}
\hline DIA & $\begin{array}{c}\text { ASPECTO } \\
\text { EVALUADO }\end{array}$ & EVALUADOR & INSTRUMENTO \\
\hline LUNES & Trabajo cooperativo & Estudiantes & cuestionario \\
\hline MARTES & Informe avance & docentes & Rúbrica \\
\hline MIERCOLES & Trabajo cooperativo & estudiantes & Cuestionario \\
\hline JUEVES & Informe de avance & docentes & Rúbrica \\
\hline \multirow{2}{*}{ VIERNES } & Informe de avance & docentes & Rúbrica \\
\cline { 2 - 4 } & Trabajo individual & estudiantes & Pauta de cotejo \\
\hline LUNES & Informe final & docentes & Rúbrica \\
\hline VIERNES & Presentación & docentes & $\begin{array}{c}\text { Pauta de } \\
\text { desempeño }\end{array}$ \\
\hline
\end{tabular}


Como se observa en la Tabla 3, el proceso de evaluación se centró principalmente en dos aspectos: los productos del trabajo de los estudiantes y la dinámica de trabajo cooperativo. Para el cálculo de la calificación final se excluyeron las evaluaciones realizadas a los informes de avance, ya que estas se utilizaron para orientar el trabajo de los estudiantes, así como también las evaluaciones del trabajo cooperativo. Esto responde a la lógica del método ABP, en la cual los logros parciales y la dinámica de funcionamiento grupal reportan al necesario proceso de retroalimentación que requieren los estudiantes para culminar exitosamente el proceso. De esta forma, se puede señalar que la evaluación formativa (formal o informal) se constituyó en una actividad transversal al desarrollo del proyecto.

Adicionalmente, las evaluaciones realizadas por los estudiantes del trabajo cooperativo permiten tener una aproximación a la percepción respecto del trabajo realizado, con el objeto de establecer modificaciones para una posterior implementación en las asignaturas de especialidad.

Para la obtención de la calificación final del ABP se utilizó los resultados obtenidos del informe y presentación final, así como también de la heteroevaluación. De esta forma se obtiene una nota final ponderada como se establece en la Tabla 3:

Tabla 3. Calculo Calificación Final ABP

\begin{tabular}{|c|c|c|}
\hline Informe Final & Presentación & Heteroevaluación \\
\hline $50 \%$ & $20 \%$ & $30 \%$ \\
\hline
\end{tabular}

\subsection{INSTRUMENTOS}

\subsubsection{Autoevaluación}

Se diseñaron tres cuestionarios con preguntas abiertas para ser autoadministrados y orientados a evaluar trabajo cooperativo.

Cuestionario 1: De aplicación el día lunes, cuyo objetivo es evaluar el trabajo cooperativo. Está compuesto por 7 ítems de pregunta abierta que indagan en relación a dificultades, estrategias de resolución, aprendizajes y apoyos y una matriz de $2 \mathrm{x} 2$ para identificar fortalezas y debilidades. Para su implementación se separa al grupo en dos subgrupos cada uno de los cuales procede a realizar la evaluación.

Cuestionario 2: De aplicación el día miércoles, cuyo objetivo se centra en el trabajo cooperativo. Se establece como seguimiento a la evolución del grupo y está compuesto por 4 ítems de pregunta abierta centradas en la identificación de dificultades y estrategias de solución implementadas por los estudiantes. Para su implementación se separa al grupo en dos subgrupos cada uno de los cuales procede a realizar la evaluación.

Cuestionario 3: De aplicación el día viernes, cuyo objetivo es evaluar el desempeño individual de los estudiantes y su aporte al trabajo cooperativo. Está compuesto por 7 ítems de pregunta abierta y es completada individualmente por cada integrante de los grupos de trabajo. 


\subsubsection{Heteroevaluación}

Se utilizó una lista de cotejo compuesta por 17 afirmaciones referidas al desempeño y actitudes de cada uno de los integrantes de los grupos. Esta evaluación fue realizada por el líder y secretarios. La evaluación del líder fue hecha por los secretarios y, a su vez, el líder evaluó a los secretarios.

\subsubsection{Evaluación Informe Final}

Se diseñó una rúbrica de desempeño compuesta por 12 criterios o aspectos a evaluar en el informe, cada uno de los cuales se desglosa en cuatro niveles de desempeño que se valoran en una escala de 4 a 1 puntos, obteniéndose un puntaje total de 48 puntos. Esta evaluación es realizada por dos docentes de planta de la carrera, siendo de esta forma la calificación final de cada grupo el promedio de las evaluaciones realizadas por los docentes.

\subsubsection{Evaluación Exposición Final}

Se diseñó una lista de cotejo compuesta por cuatro criterios a evaluar que son claridad, coherencia, contenido y exposición. Los tres primeros se desagregan en 3 indicadores y el último en 6. Cada indicador se valora en una escala tipo Likert según la Figura 6. De esta forma, se obtiene un puntaje total de 75 puntos.

Figura 5. Escala de Valoración Exposiciones

\begin{tabular}{|c|c|}
\hline CRITERIO & VALORACIÓN \\
\hline Siempre & 5 \\
\hline Casi Siempre & 4 \\
\hline A veces & 3 \\
\hline Rara vez & 2 \\
\hline No observado & 1 \\
\hline
\end{tabular}

\subsubsection{Evaluación Informes de Avance}

Para evaluar los reportes parciales (identificación del problema, formulación de hipótesis y pre informe final) de los estudiantes se diseñaron tres rúbricas de desempeño, cuyo objetivo es retroalimentar a los grupos. Cada una de estas rúbricas está compuesta por 5 criterios o aspectos a evaluar, cada uno de los cuales se valora en cuatro niveles de desempeño.

\subsubsection{Evaluación del Portafolio}

Se diseñó una lista de cotejo que definía y evaluaba la reflexión y organización de todas las evidencias que informaban sobre todas las etapas del proceso. Se destaca en esta evaluación las conclusiones como una instancia de reflexión que busca el análisis profundo del proceso vivido. 


\section{RESULTADOS OBTENIDOS}

A continuación, se presenta el resumen de las calificaciones promedio obtenidas por cada uno de los grupos (Tabla 4):

Tabla 4. Resumen de calificaciones por Grupo

\begin{tabular}{|c|c|c|c|c|}
\hline GRUPO & EXPOSICIÓN & INFORME & HETEROEVALUACIÓN & NOTA FINAL \\
\hline 1 & 5.1 & 6.5 & 5.5 & 5.9 \\
\hline 2 & 7.0 & 5.9 & 5.3 & 5.9 \\
\hline 3 & 6.3 & 6.2 & 6.3 & 6.3 \\
\hline 4 & 6.7 & 6.5 & 5.6 & 6.3 \\
\hline 5 & 6.5 & 5.8 & 6.9 & 6.3 \\
\hline 6 & 6.3 & 5.9 & 6.2 & 6.1 \\
\hline 7 & 5.5 & 6.2 & 6.1 & 6.0 \\
\hline 8 & 6.2 & 6.8 & 7.0 & 6.7 \\
\hline 9 & 6.5 & 6.1 & 6.6 & 6.3 \\
\hline 10 & 6.2 & 6.4 & 5.7 & 6.1 \\
\hline
\end{tabular}

El análisis de las calificaciones obtenidas por los estudiantes se presenta en la siguiente tabla resumen (Tabla 5):

Tabla 5. Resumen Resultados ABP

\begin{tabular}{|c|c|c|c|c|c|c|}
\hline & $\mathrm{N}$ & Mínimo & Máximo & Media & Desv. típ. & Varianza \\
\hline Exposición & 110 & 5.1 & 7.0 & 6.230 & .5298 & .281 \\
\hline Informe & 110 & 5.8 & 6.8 & 6.230 & .3049 & .093 \\
\hline Heteroevaluación & 110 & 1.0 & 7.0 & 6.124 & 1.3674 & 1.870 \\
\hline Final ponderado & 110 & 4.6 & 6.7 & 6.198 & .4381 & .192 \\
\hline N válido (según lista) & 110 & & & & & \\
\hline
\end{tabular}

Los resultados obtenidos por los estudiantes en las distintas evaluaciones tienden al valor 6.2. Sin embargo, se presenta una mayor dispersión de puntajes en la Heteroevaluación y Evaluación de las exposiciones finales $(\mathrm{DS}=1.37$; $\mathrm{DS}=0.53)$ en comparación a la evaluación del informe final (DS=0.30).

Considerando los resultados por tipo de evaluación, se puede señalar que, en el informe final, el $70 \%$ de los estudiantes obtiene una calificación mayor o igual a 6.0. El 30\% restante se sitúa con calificaciones entre 5.8 y 5.9. A su vez, en la evaluación de las exposiciones finales, el $80 \%$ de los estudiantes obtiene calificaciones mayores o iguales a 6.0 y el $20 \%$ restante se sitúa entre el 5.1 y 5.9 .

Las mayores diferencias se observan en la heteroevaluación donde el $8 \%$ de los estudiantes obtiene una calificación inferior a 4.0; el 19\% obtiene calificaciones que se 
encuentran en el rango 4.0 - 5.7 y el $73 \%$ restante alcanza calificaciones por sobre el 6.0, observándose que el $48 \%$ de los estudiante alcanza la calificación 7.0 en este tipo de evaluación.

Estas diferencias se pueden explicar en razón a las características de los distintos tipos de evaluación. En este sentido, la evaluación del informe y exposición corresponden a evaluaciones del producto final de cada uno de los grupos, siendo esperable que se enmascaren diferencias individuales en los desempeños.

Adicionalmente, en las exposiciones, las diferencias entre los grupos estarían asociadas con las habilidades como expositores de los estudiantes que realizaron la presentación, por tanto, la calificación recoge, al menos parcialmente, estas diferencias.

En cuanto a la heteroevaluación, esta entrega mayor información respecto de los desempeños individuales, por consiguiente, era esperable una mayor dispersión de puntajes. Estas evaluaciones dan cuenta de la percepción del grupo en relación a los aportes de cada uno de los integrantes, observándose la existencia de sanciones a los estudiantes que no cumplieron con las tareas asignadas o cuyos aportes eran percibidos como deficientes en comparación a lo esperado.

En relación a las evaluaciones del proceso, se observa que los estudiantes identifican como principales dificultades en el desarrollo del proyecto la organización y cumplimiento de compromisos de parte de los integrantes del grupo, la falta de interés y desmotivación, así como también falta de espacios físicos adecuados para el trabajo del grupo y la coordinación entre los docentes en el proceso de guía.

Estas dificultades son identificadas como debilidades significativas, especialmente al inicio del proyecto, pero que disminuyen en relevancia a medida que se avanzaba en el trabajo. Esto se asocia a acciones específicas implementadas para superar estos obstáculos, como, por ejemplo, subdividir el grupo; establecimiento de metas a corto y mediano plazo; gestión de recursos (solicitud de salas en biblioteca y aulas en horarios alternos a los de clases) y optar por seguir las orientaciones solo de uno de los docentes, entre otras.

Como fortalezas se identifican aspectos relacionados a las relaciones al interior del grupo como respeto, tolerancia, coordinación y comunicación. En este sentido, si bien se reconocen la existencia de problemas, los estudiantes valoran el trabajo de equipo y la forma en que coordinaron los esfuerzos individuales en función de un objetivo común.

Los estudiantes concuerdan en señalar que la metodología utilizada en el proyecto les ha permitido aprender una estrategia para la resolución de problemas y adquisición de aprendizaje de contenidos de temas relacionados con educación. Además, identifican el aporte del proyecto en el trabajo de equipo que, si bien no indican el aspecto específico, reconocen mejoras en las relaciones interpersonales y en la organización del trabajo.

A su vez, los estudiantes consideran que los contenidos de las asignaturas constituyen un aporte a la identificación del problema, así como también al proceso de elaboración de una estrategia de solución, aun cuando en la información entregada no se identifica un contenido o asignatura en particular.

Respecto del acompañamiento de parte de los docentes, los estudiantes evalúan positivamente este proceso. Sin embargo, señalan dificultades asociadas a las diferencias existentes en la visión que los docentes les presentan del caso y/o del problema que han identificado. Esta situación si bien es señalada como un problema para los estudiantes generó asumir acciones grupales como tomar decisiones con respecto a cuál sería la perspectiva del análisis y posterior solución que asumirían. 
Desde la percepción de los docentes, la implementación del proyecto tuvo resultados positivos, destacando el compromiso asumido por los estudiantes para el logro del objetivo final, el cumplimiento con los plazos establecidos, la información recogida que sustentaba el análisis, la organización de los grupos reflejada en la entrega de información y aportes durante las sesiones tutoriales, y la calidad del informe final.

Finaliza el trabajo con la presentación oral del informe donde cada grupo se organiza designando uno o dos expositores que exponen el trabajo realizado. Se entregan los portafolios, que, además, de contener las evaluaciones y bitácoras de trabajo, reportan información sobre el proceso de recogida, análisis y selección de información proveniente de diferentes fuentes.

\section{CONCLUSIONES}

El Proyecto de Aprendizaje Basado en Problemas busca desarrollar en los estudiantes de pregrado competencias personales relacionadas con habilidades sociales y competencias profesionales vinculadas con la capacidad de resolver problemas. Por medio de la identificación de conceptos claves, se espera que estas competencias orienten la búsqueda de soluciones basadas en elementos teóricos en contexto de equipos de trabajo, cuya organización permitirá el éxito final. Promueve el conocimiento, el razonamiento, la comunicación y la toma de decisiones a través de una metodología con una estructura que obliga a la búsqueda, selección y análisis de la información. Esta situación le permite enfrentar la necesidad de conducir sus propios aprendizajes e ir adquiriendo un conocimiento integrador y crítico que se podrá incrementar a través de las aplicaciones sucesivas de la forma de trabajo, mediante las interacciones socializantes del trabajo en un ambiente colaborativo (Pezoa \& Labra, 2000).

Desde esta perspectiva, la carrera de Pedagogía en Educación Diferencial diseña el proyecto para su implementación en los estudiantes de $1^{\circ}$ año y su posterior aplicación en asignaturas disciplinares, "favoreciendo, de esta forma, el desarrollo de habilidades y destrezas sociales, actitudinales y cognitivas para la búsqueda, análisis integración y transferencia de conocimientos" (objetivo general) para llegar a la solución de problemas. Para el logro de este objetivo, se utiliza el estudio de caso que permite plantear una situación real con variables que deben ser relacionadas para comprender la situación problemática y organizar desde su análisis la búsqueda de soluciones. El docente asume el rol de facilitador que orienta, en sus diferentes etapas, la reflexión, discusión, valoración, selección y toma de decisiones del grupo.

En el proceso de implementación del proyecto se obtuvieron como productos: el informe final de cada uno de los grupos, así como también el registro en video de las exposiciones realizadas por los estudiantes. De los resultados obtenidos del proceso de evaluación se puede concluir que los estudiantes alcanzaron un nivel de desempeño acorde a los objetivos del proyecto. Esto en base a las calificaciones finales ponderadas ( $\mathrm{ME}=6.2, \mathrm{DS}=0.44)$, las cuales dan cuenta de un buen desarrollo de la metodología ABP. Además, los estudiantes concuerdan al señalar que lograron conocer y aplicar una estrategia general de resolución de problemas.

Asociado a la implementación del proyecto, los estudiantes reconocen el impacto de la metodología en la dinámica de trabajo, siendo frecuente el identificar aspectos tales 
como la coordinación y organización del grupo en calidad de aprendizaje que tuvo lugar durante el desarrollo del proyecto.

Las calificaciones obtenidas evidencian una importante dispersión de notas en la heteroevaluación ( $\mathrm{ME}=6.12 \mathrm{DS}=1,36)$, con una disminución para la evaluación del informe $(\mathrm{ME}=6.23 \mathrm{DS}=0.30)$ y la exposición final $(\mathrm{ME}=6.23 \mathrm{DS}=0.53)$. Esto puede explicarse a partir del valor asignado por los estudiantes a factores como el compromiso, motivación y calidad de los aportes de cada uno de los integrantes al producto final. Paralelamente, este tipo de evaluación, por sus características, no enmascara diferencias individuales como ocurre con las calificaciones del informe y exposición final del proyecto.

Al considerar la proporción de estudiantes con calificaciones por debajo de la media, se observa que esta es del $20 \%$ para el informe, $40 \%$ para la exposición y del $36 \%$ para la heteroevaluación. Se revela que, solo para este último tipo de evaluación, hay estudiantes que se encuentran por debajo de la calificación mínima de aprobación (8\%). De esta forma, se puede señalar que los resultados indican que las metas propuestas para el proyecto se lograron.

El Método de Aprendizaje Basado en Problemas constituye una herramienta de aprendizaje efectiva para crear espacios para el diálogo, la tolerancia y la búsqueda de soluciones, lo que promueve el conocimiento, el razonamiento, la comunicación y la toma de decisiones en un trabajo en equipo. Esto se evidencia en los comentarios realizados por los estudiantes y por las observaciones realizadas por los docentes. En ambos casos, se establece que los grupos implementaron distintas vías para el afrontamiento efectivo del caso-problema, así como también de las dificultades que se presentaron en su funcionamiento como grupo.

La utilización de la metodología ABP favorece la integración, transferencia y aplicabilidad del conocimiento. Además, estimula a los estudiantes hacia la acción y el trabajo independiente. Constituye una actividad integradora que permite relacionar experiencias previas con las actuales, integrando contenidos y disciplinas del currículum, promoviendo la búsqueda y reflexión.

En este sentido, las acciones realizadas por los estudiantes más la guía de los docentes permite establecer relaciones entre contenidos de las distintas asignaturas cursadas. Adicionalmente, estimula la recopilación de información y, por tanto, el desarrollo de estrategias para la búsqueda y selección de contenidos en función de una problemática específica. De esta forma, los casos-problema se constituyen en el contexto para que se estimule el desarrollo de habilidades y competencias cognitivas.

Al enfrentar los casos, los estudiantes ven favorecido su aprendizaje al facilitar la comprensión de contenidos que deben ser aplicados en un contexto específico. De esta forma, se promueve el logro de aprendizajes significativos en una actividad que se enmarca en los lineamientos que señala el aprendizaje por descubrimiento significativo. Además, en consideración al rol que cumplen los docentes se establece un proceso que presenta características del descubrimiento guiado y descubrimiento autónomo (Arancibia, Herrera \& Strasser, 2005).

Los docentes establecen orientaciones generales a partir del trabajo realizado por los estudiantes; no entregan respuestas o inducen a un determinado tipo de respuesta, su rol se limita a canalizar los esfuerzos de los grupos y cumplen una función facilitadora. A su vez, cada grupo establece tareas y acciones para el logro de las mismas, las cuales requieren de un proceso consciente de toma de decisiones. Este proceso y las estrategias 
utilizadas implican aprender sobre sí mismos y sobre la dinámica de interacción social, todo lo cual permite dar un sentido único al proceso de aprendizaje.

En el desarrollo e implementación del proyecto se evidenció la necesidad de incorporar modificaciones para futuras aplicaciones. En este sentido, los estudiantes señalan que la rotación de docentes en el proceso de guía generaba confusiones, lo cual se explica en función de las diversas perspectivas que se adoptaban para enfrentar el caso-problema. En este sentido, si bien la rotación responde a una dificultad practica $\left(\mathrm{n}^{\mathrm{o}}\right.$ de docentes de planta), se vería favorecido el proceso de aprendizaje de los estudiantes al ser orientados por un solo docente durante todo el proceso, teniendo la posibilidad de consultar a otros profesores cuando el grupo lo estime conveniente. Esto tendría un impacto positivo en la organización y coordinación, además de permitir optimizar los tiempos de trabajo en aula, lo que favorecería la retroalimentación y la calidad de los trabajos presentados por los distintos grupos.

En el proceso de acompañamiento es necesaria la revisión del estado de avance del trabajo de los estudiantes. De esta forma, se requiere de la implementación del 100\% del cronograma de actividades. Esto requiere de ajustar las actividades en base a lo observado en el desarrollo del proyecto, ya que actividades como la presentación oral de estados de avance no se realizaron y no todos los grupos entregaron los tres informes de avance. Esto permitiría a los grupos organizar de forma más efectiva sus tiempos, así como también facilitaría la función de guía del docente.

Al revisar las intenciones y objetivos del proyecto en relación a los resultados obtenidos, se evidencia un vacío de información referente a las competencias y/o habilidades desarrolladas por los estudiantes. Para esto, se requiere de la utilización de un procedimiento pre-pos test que evalúe las competencias de entrada de los alumnos y el nivel alcanzado por las mismas con posterioridad al desarrollo del ABP. Esto requiere de la utilización de un instrumento que sea válido y confiable en la medición de las competencias que, desde lo teórico, se asocian a la resolución de problemas.

En cuanto a los instrumentos utilizados, los resultados arrojados no dan cuenta de las estrategias específicas utilizadas para la toma de decisiones, búsqueda y selección de información, organización del grupo, entre otras. Para esto, se deben adecuar los instrumentos, incluyendo ítems que permitan a los estudiantes detallar la forma en que realizaron el trabajo y/o enfrentaron los problemas. Además, se puede incorporar en los informes un apartado donde se le solicite al grupo detallar las distintas etapas, acciones y estrategias utilizadas para la conformación del producto final que es el informe.

No cabe duda que analizando las diferentes evaluaciones aplicadas y los productos que durante el proceso y al final de este se presentaron, la metodología de Aprendizaje Basado en Problema constituye una herramienta útil para el desarrollo de habilidades cognitivas y sociales y una estrategia que enseña un camino para la adquisición de nuevos aprendizajes, dándole un cierto nivel de autonomía. Estas características deberían incrementarse a través de la aplicación sistemática en diferentes asignaturas de la carrera.

\section{REFERENCIAS BIBLIOGRÁFICAS}

Arancibia, V., Herrera, P. y Strasser, K. (2005). Manual de Psicología Educacional. Santiago de Chile: Editorial Universidad Católica. 
Arends, R. (2007). Aprender a Enseñar. $7^{\circ}$ edición. México: McGraw-Hill.

Coll, C., Mauri, T y Onrubia, J. (2006). Análisis y Resolución de Caso-Problema Mediante el Aprendizaje Colaborativo. Revista de Universidad y Sociedad del Conocimiento, 3(2). Extraído http://www.uoc.edu/rusc/3/2/dt/esp/coll_mauri_onrubia.pdf

Kingler, C. y Vadillo, G. (2003). Psicología Cognitiva. México: McGraw-Hill.

Mucchielli, R. (1970). La dinámica de los grupos. Madrid: Ibérica Europea de Ediciones.

Slavin, R. (2008). Aprendizaje Cooperativo, "Éxito Para Todos" y Reforma Basada en la Evidencia Para Todos. Ponencia Congreso Anual Sobre Fracaso Escolar. Extraído http://www.fracasoescolar.com/conclusions2008/rslavin.pdf

Pezoa, C. y Labra, J. (2000). Las estrategias de Aprendizaje, una propuesta en el Contexto Universitario. Serie Material de Apoyo a la Docencia. Santiago de Chile: Universidad Católica Cardenal Raúl Silva Henríquez.

Woolfolk, A. (2006). Psicología Educativa. $9^{\circ}$ edición. Ohio: Pearson 
